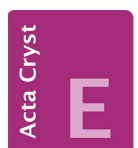

CRYSTALLOGRAPHIC COMMUNICATIONS

ISSN 2056-9890

Received 3 September 2015

Accepted 11 September 2015

Edited by H. Stoeckli-Evans, University of Neuchâtel, Switzerland

Keywords: crystal structure; 3-methylpyridinium picrate; triclinic polymorph; $\pi-\pi$ stacking; anticonvulsant activity.

CCDC reference: 1417794

Supporting information: this article has supporting information at journals.iucr.org/e

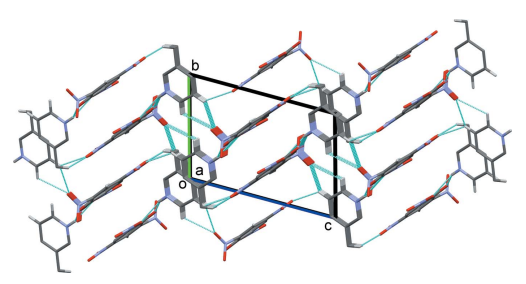

\section{Crystal structure of 3-methylpyridinium picrate: a triclinic polymorph}

\author{
Jeganathan Gomathi and Doraisamyraja Kalaivani*
}

PG and Research Department of Chemistry, Seethalakshmi Ramaswami College, Tiruchirappalli 620 002, Tamil Nadu, India. *Correspondence e-mail: kalaivbalaj@yahoo.co.in

The title molecular salt, $\mathrm{C}_{6} \mathrm{H}_{8} \mathrm{~N}^{+} \cdot \mathrm{C}_{6} \mathrm{H}_{2} \mathrm{~N}_{3} \mathrm{O}_{7}^{-}$(systematic name: 3-methylpyridinium 2,4,6-trinitrophenolate), crystallizes in the triclinic space group $P \overline{1}$. The crystal structure of the monoclinic polymorph (space group $P 2_{1} / n$ ) has been reported [Stilinovic \& Kaitner (2011). Cryst. Growth Des. 11, 4110-4119]. In the crystal, the anion and cation are linked via bifurcated $\mathrm{N}-\mathrm{H} \cdots(\mathrm{O}, \mathrm{O})$ hydrogen bonds, enclosing an $R_{1}^{2}(6)$ graph-set motif. These units are linked via $\mathrm{C}-\mathrm{H} \cdots \mathrm{O}$ hydrogen bonds, forming a three-dimensional framework. Within the framework there are $\pi-\pi$ interactions present, involving inversion-related picrate anions and inversion-related pyridinium cations, with inter-centroid distances of 3.7389 (14) and 3.560 (2) , respectively.

\section{Chemical context}

Stilinovic \& Kaitner (2011) have synthesized a series of 20 crystalline picrates of pyridine derivatives and through single crystal X-ray diffraction studies revealed the presence of a common synthon. They reported the crystal structure of the monoclinic polymorph of the title molecular salt: space group $P 2_{1} / n$.<smiles></smiles>

The observation that the presence of more than one heterocyclic component in a molecule enhances the biological response and thermal stability encouraged us to synthesize several new carbon-bonded anionic sigma complexes from chloronitro-aromatic compounds and pyrimidine derivatives in the presence of pyridine bases (Babykala et al., 2014; Buvaneswari \& Kalaivani, 2013; Mangaiyarkarasi \& Kalaivani, 2013; Manickkam \& Kalaivani, 2011, 2014; Sridevi \& Kalaivani, 2012). Surprisingly, when we made an attempt to synthesize a similar type of complex from the electrondeficient chloronitroaromatic compound (picryl chloride), an imidazole derivative (hydantoin) and 3-methylpyridine, the title salt crystallized from the medium (ethanol) instead of the expected carbon-bonded anionic sigma complex.

\section{Structural commentary}

The molecular structure of the title molecular salt is shown in Fig. 1. The anion and cation are linked via bifurcated $\mathrm{N}-$ 


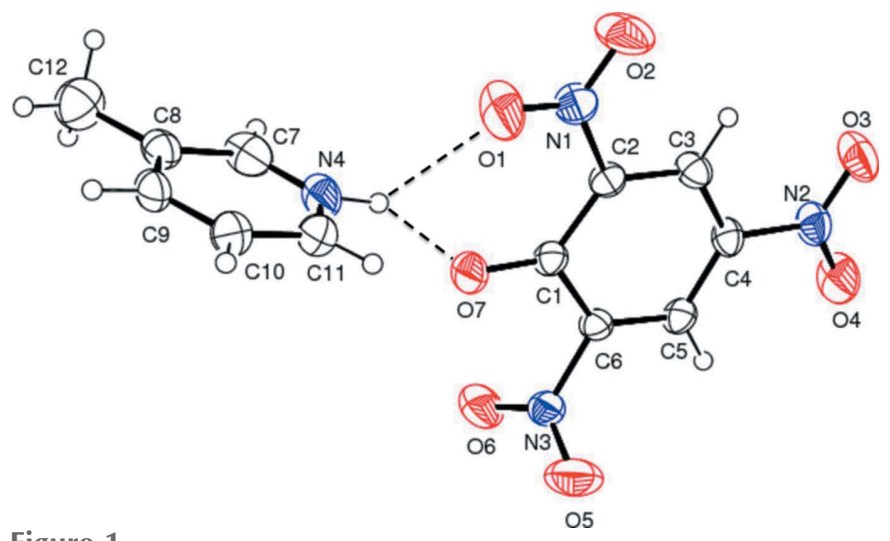

Figure 1

A view of the molecular structure of the title molecular salt, with atom labelling. Displacement ellipsoids are drawn at the $40 \%$ probability level. Hydrogen bonds are shown as dashed lines (see Table 1).

$\mathrm{H} \cdots(\mathrm{O}, \mathrm{O})$ hydrogen bonds, enclosing an $R_{1}^{2}(6)$ graph-set motif (Fig. 1 and Table 1 ). In the picrate anion, the two nitro groups flanking the $\mathrm{C}-\mathrm{O}^{-}$bond are oriented differently. Nitro group $\mathrm{O} 1 / \mathrm{N} 1 / \mathrm{O} 2$, involved in $\mathrm{N}-\mathrm{H} \cdots \mathrm{O}$ hydrogen bonds as noted above, is inclined to the benzene ring by $6.7(3)^{\circ}$, while nitro group $\mathrm{O} 5 / \mathrm{N} 3 / \mathrm{O} 6$ is inclined to the benzene ring by $70.07(3)^{\circ}$, probably to alleviate steric crowding. The third nitro group $(\mathrm{O} 3 / \mathrm{N} 2 / \mathrm{O} 4)$, para with respect to the $\mathrm{C}-\mathrm{O}^{-}$ bond, deviates only slightly from the benzene ring, making a dihedral angle of $6.6(3)^{\circ}$.

\section{Supramolecular features}

In the crystal, the anionic and cationic hydrogen-bonded units are linked via $\mathrm{C}-\mathrm{H} \cdots \mathrm{O}$ hydrogen bonds, forming a three-

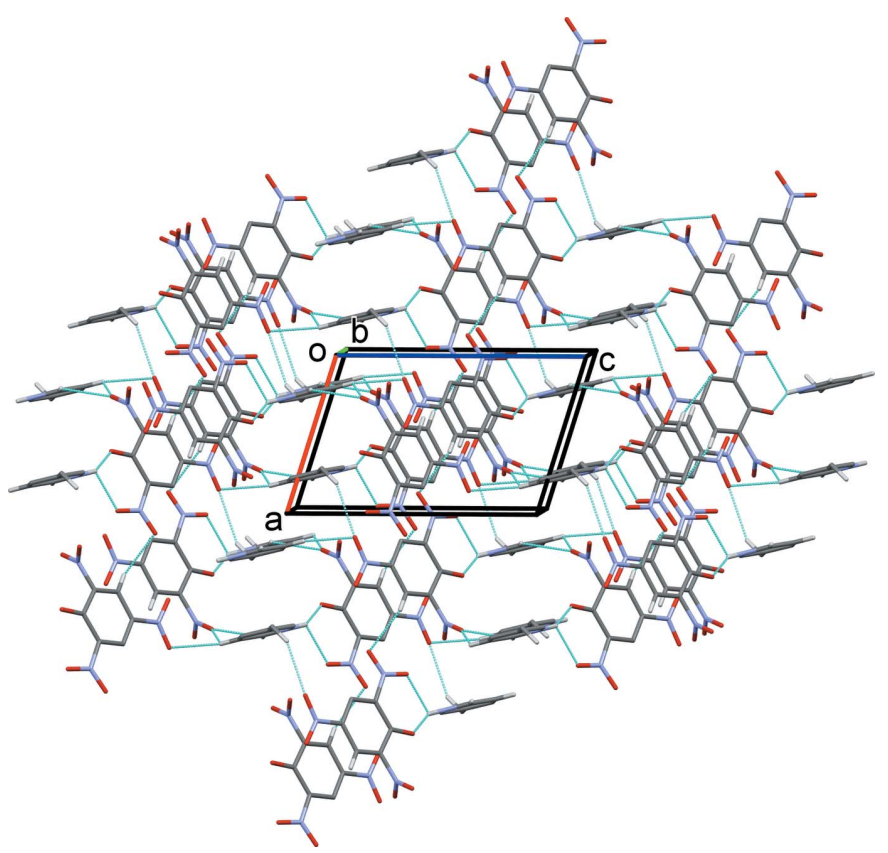

Figure 2

A view along the $b$ axis of the crystal packing of the title molecular salt. Hydrogen bonds are shown as dashed lines (see Table 1), and $\mathrm{H}$ atoms not involved in these interactions have been omitted for clarity.
Table 1

Hydrogen-bond geometry $\left(\AA,^{\circ}\right)$.

\begin{tabular}{lllll}
\hline$D-\mathrm{H} \cdots A$ & $D-\mathrm{H}$ & $\mathrm{H} \cdots A$ & $D \cdots A$ & $D-\mathrm{H} \cdots A$ \\
\hline $\mathrm{N} 4-\mathrm{H} 4 A \cdots \mathrm{O} 1$ & $0.93(4)$ & $2.27(4)$ & $2.827(4)$ & $118(4)$ \\
N4-H4A $\cdots \mathrm{O} 7$ & $0.93(4)$ & $1.79(5)$ & $2.638(4)$ & $152(4)$ \\
$\mathrm{C} 5-\mathrm{H} 5 \cdots 2^{\mathrm{i}}$ & 0.93 & 2.51 & $3.406(4)$ & 162 \\
$\mathrm{C} 10-\mathrm{H} 10 \cdots \mathrm{O}^{\mathrm{ii}}$ & 0.93 & 2.55 & $3.220(4)$ & 129 \\
$\mathrm{C}^{\mathrm{i}}-\mathrm{H} 12 B \cdots 3^{\mathrm{iii}}$ & 0.96 & 2.56 & $3.414(5)$ & 149 \\
\hline
\end{tabular}

Symmetry codes: (i) $x-1, y, z$; (ii) $x, y-1, z-1$; (iii) $-x+2,-y+1,-z+1$.

dimensional structure (Figs. 2 and 3, and Table 1). Within this framework there are slipped parallel $\pi-\pi$ interactions present, involving inversion-related picrate anions [inter-centroid distance $=3.7389$ (14) $\AA$, inter-planar distance = 3.5829 (8) $\AA$, slippage $=1.069 \AA$ ] and inversion-related pyridinium cations [inter-centroid distance $=3.560(2) \AA$, inter-planar distance $=$

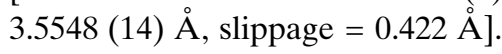

\section{Anticonvulsant activity}

The anticonvulsant activity of synthesized 3-methylpyridinium picrate has been measured by employing the maximal electro shock (MES) method (Bhattacharya \& Chakrabarti, 1998; Misra et al., 1973). Different stages of convulsion such as tonic flexion, tonic extensor, clonus convulsion, stupor and recovery/death have been examined. Though all phases are reduced, noticeable decrease is observed in the clonus phase and hence the title molecule may be a potent agent for controlling myoclonic type epilepsy in the future.

\section{Database survey}

A search of the Cambridge Structural Database (Version 5.36, last update May 2015; Groom \& Allen, 2014) yielded 40 hits for meta- or para-substituted pyridinium picrate salts. In the picrate anions, the average dihedral angle of the nitro group para to the $\mathrm{C}-\mathrm{O}^{-}$bond with respect to the benzene ring is $\mathrm{ca}$ $6^{\circ}$, while for the two nitro groups on either side of the $\mathrm{C}-\mathrm{O}^{-}$ bond the dihedral angles are both $c a$ 26-28. In the title

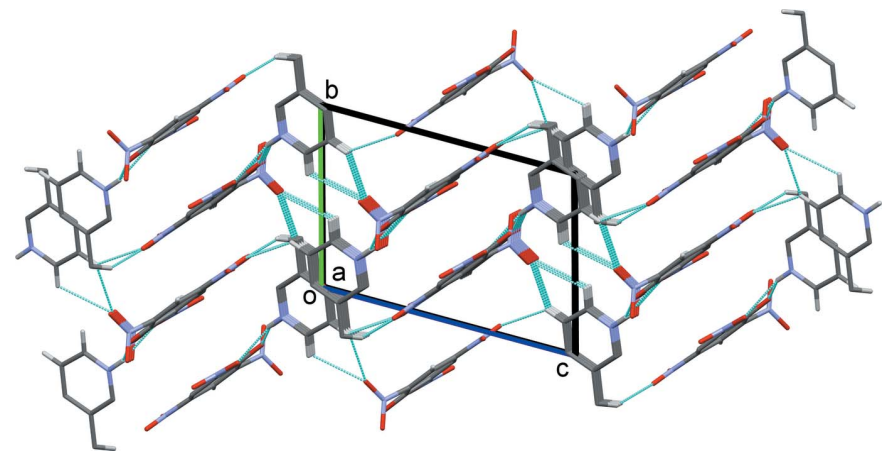

Figure 3

A view along the $a$ axis of the crystal packing of the title molecular salt. Hydrogen bonds are shown as dashed lines (see Table 1), and $\mathrm{H}$ atoms not involved in these interactions have been omitted for clarity. 
Table 2

Experimental details.

\begin{tabular}{|c|c|}
\hline \multicolumn{2}{|l|}{ Crystal data } \\
\hline Chemical formula & $\mathrm{C}_{6} \mathrm{H}_{8} \mathrm{~N}^{+} \cdot \mathrm{C}_{6} \mathrm{H}_{2} \mathrm{~N}_{3} \mathrm{O}_{7}^{-}$ \\
\hline$M_{\mathrm{r}}$ & 322.24 \\
\hline Crystal system, space group & Triclinic, $P \overline{1}$ \\
\hline Temperature $(\mathrm{K})$ & 293 \\
\hline$a, b, c(\AA)$ & $8.1224(5), 8.7016(5), 11.3161(6)$ \\
\hline$\alpha, \beta, \gamma\left({ }^{\circ}\right)$ & $98.239(3), 100.318(3), 117.635(3)$ \\
\hline$V\left(\AA^{3}\right)$ & $673.17(7)$ \\
\hline$Z$ & 2 \\
\hline Radiation type & Mo $K \alpha$ \\
\hline$\mu\left(\mathrm{mm}^{-1}\right)$ & 0.13 \\
\hline Crystal size (mm) & $0.35 \times 0.30 \times 0.25$ \\
\hline \multicolumn{2}{|l|}{ Data collection } \\
\hline Diffractometer & Bruker Kappa APEXII CCD \\
\hline Absorption correction & $\begin{array}{l}\text { Multi-scan (SADABS; Bruker, } \\
\text { 2004) }\end{array}$ \\
\hline$T_{\min }, T_{\max }$ & $0.952,0.969$ \\
\hline $\begin{array}{l}\text { No. of measured, independent and } \\
\text { observed }[I>2 \sigma(I)] \text { reflections }\end{array}$ & $13299,2374,1717$ \\
\hline$R_{\text {int }}$ & 0.029 \\
\hline$(\sin \theta / \lambda)_{\max }\left(\AA^{-1}\right)$ & 0.595 \\
\hline \multicolumn{2}{|l|}{ Refinement } \\
\hline$R\left[F^{2}>2 \sigma\left(F^{2}\right)\right], w R\left(F^{2}\right), S$ & $0.045,0.131,1.07$ \\
\hline No. of reflections & 2374 \\
\hline No. of parameters & 212 \\
\hline $\mathrm{H}$-atom treatment & $\begin{array}{l}\mathrm{H} \text { atoms treated by a mixture of } \\
\text { independent and constrained } \\
\text { refinement }\end{array}$ \\
\hline$\Delta \rho_{\max }, \Delta \rho_{\min }\left(\mathrm{e} \AA^{-3}\right)$ & $0.24,-0.25$ \\
\hline
\end{tabular}

Computer programs: APEX2, SAINT and XPREP (Bruker, 2004), SIR92 (Altomare et al., 1993), ORTEP-3 for Windows (Farrugia, 2012), Mercury (Macrae et al., 2008), SHELXL2014 (Sheldrick, 2015) and PLATON (Spek, 2009).

compound, the latter two dihedral angles are quite different being 6.7 (3) and $70.07(3)^{\circ}$. In the monoclinic polymorph (UBEFEO; Stilinovic \& Kaitner, 2011), these three dihedal angle are $c a 3.60,6.92$ and $13.83^{\circ}$, respectively, and the cation and anion are also linked via bifurcated $\mathrm{N}-\mathrm{H} \cdots(\mathrm{O}, \mathrm{O})$ hydrogen bonds.

\section{Synthesis and crystallization}

Picryl chloride $[2.56 \mathrm{~g}(0.01 \mathrm{~mol})]$ was dissolved in $30 \mathrm{ml}$ of rectified spirit and mixed with hydantoin $[1.00 \mathrm{~g}(0.01 \mathrm{~mol})]$ in $30 \mathrm{ml}$ of rectified spirit. After mixing these solutions, $3 \mathrm{ml}$ of 3 -methylpyridine $(0.03 \mathrm{~mol})$ was added and the temperature of the mixture was raised to $313 \mathrm{~K}$. At this temperature, the mixture was stirred for 5 to $6 \mathrm{~h}$. The solution was then cooled to room temperature, filtered and the filtrate kept at $298 \mathrm{~K}$. After a period of 4 weeks, dark shiny maroon-red-coloured crystals formed from the solution. The crystals were filtered, powdered and dried. The dry solid was washed with $50 \mathrm{ml}$ of dry ether ( $5 \mathrm{ml}$ for each aliquot) and recrystallized from rectified spirit (yield: 60\%; m.p. $483 \mathrm{~K}$ ).

\section{Refinement}

Crystal data, data collection and structure refinement details are summarized in Table 2. The NH H atom was located in a difference Fourier map and freely refined. The C-bound $\mathrm{H}$ atoms were included in calculated positions and refined as riding: $\mathrm{C}-\mathrm{H}=0.93-0.96 \AA$ with $U_{\text {iso }}(\mathrm{H})=1.2 U_{\text {eq }}(\mathrm{C})$.

\section{Acknowledgements}

The authors are thankful to the DST-SERB, New Delhi, for financial support and the SAIF, IIT Madras, Chennai, for the single crystal XRD data collection.

\section{References}

Altomare, A., Cascarano, G., Giacovazzo, C. \& Guagliardi, A. (1993). J. Appl. Cryst. 26, 343-350.

Babykala, R., Rajamani, K., Muthulakshmi, S. \& Kalaivani, D. (2014). J. Chem. Crystallogr. 44, 243-254.

Bhattacharya, S. K. \& Chakrabarti, A. (1998). Ind. J. Exp. Biol. 36, 118-121.

Bruker (2004). APEX2, SAINT, XPREP and SADABS. Bruker AXS Inc., Madison, Wisconsin, USA.

Buvaneswari, M. \& Kalaivani, D. (2013). J. Chem. Crystallogr. 43, 561-567.

Farrugia, L. J. (2012). J. Appl. Cryst. 45, 849-854.

Groom, C. R. \& Allen, F. H. (2014). Angew. Chem. Int. Ed. 53, 662671.

Macrae, C. F., Bruno, I. J., Chisholm, J. A., Edgington, P. R., McCabe, P., Pidcock, E., Rodriguez-Monge, L., Taylor, R., van de Streek, J. \& Wood, P. A. (2008). J. Appl. Cryst. 41, 466-470.

Mangaiyarkarasi, G. \& Kalaivani, D. (2013). Crystallogr. Rep. 58, 1096-1102.

Manickkam, V. \& Kalaivani, D. (2011). Acta Cryst. E67, 03475.

Manickkam, V. \& Kalaivani, D. (2014). Acta Cryst. E70, 256-258.

Misra, A. K., Dandiya, P. C. \& Kulkarni, S. K. (1973). Ind. J. Pharmacol. 5, 449-450.

Sheldrick, G. M. (2015). Acta Cryst. C71, 3-8.

Spek, A. L. (2009). Acta Cryst. D65, 148-155.

Sridevi, G. \& Kalaivani, D. (2012). Acta Cryst. E68, o1044.

Stilinović, V. \& Kaitner, B. (2011). Cryst. Growth Des. 11, 4110-4119. 


\section{supporting information}

Acta Cryst. (2015). E71, 1196-1198 [doi:10.1107/S2056989015017090]

\section{Crystal structure of 3-methylpyridinium picrate: a triclinic polymorph}

\section{Jeganathan Gomathi and Doraisamyraja Kalaivani}

\section{Computing details}

Data collection: APEX2 (Bruker, 2004); cell refinement: APEX2 and SAINT (Bruker, 2004); data reduction: SAINT and XPREP (Bruker, 2004); program(s) used to solve structure: SIR92 (Altomare et al., 1993); program(s) used to refine structure: SHELXL2014 (Sheldrick, 2015); molecular graphics: ORTEP-3 for Windows (Farrugia, 2012) and Mercury (Macrae et al., 2008); software used to prepare material for publication: SHELXL2014 (Sheldrick, 2015) and PLATON (Spek, 2009).

\section{3-Methylpyridinium 2,4,6-trinitrophenolate}

Crystal data

$\mathrm{C}_{6} \mathrm{H}_{8} \mathrm{~N}^{+} \cdot \mathrm{C}_{6} \mathrm{H}_{2} \mathrm{~N}_{3} \mathrm{O}_{7}^{-}$

$M_{r}=322.24$

Triclinic, $P \overline{1}$

$a=8.1224(5) \AA$

$b=8.7016(5) \AA$

$c=11.3161(6) \AA$

$\alpha=98.239(3)^{\circ}$

$\beta=100.318(3)^{\circ}$

$\gamma=117.635(3)^{\circ}$

$V=673.17(7) \AA^{3}$

\section{Data collection}

Bruker Kappa APEXII CCD diffractometer

Radiation source: fine-focus sealed tube Graphite monochromator

$\omega$ and $\varphi$ scan

Absorption correction: multi-scan

(SADABS; Bruker, 2004)

$T_{\min }=0.952, T_{\max }=0.969$

Refinement

Refinement on $F^{2}$

Least-squares matrix: full

$R\left[F^{2}>2 \sigma\left(F^{2}\right)\right]=0.045$

$w R\left(F^{2}\right)=0.131$

$S=1.07$

2374 reflections

212 parameters

0 restraints
$Z=2$

$F(000)=332$

$D_{\mathrm{x}}=1.590 \mathrm{Mg} \mathrm{m}^{-3}$

Mo $K \alpha$ radiation, $\lambda=0.71073 \AA$

Cell parameters from 5179 reflections

$\theta=2.7-26.9^{\circ}$

$\mu=0.13 \mathrm{~mm}^{-1}$

$T=293 \mathrm{~K}$

Block, red

$0.35 \times 0.30 \times 0.25 \mathrm{~mm}$

13299 measured reflections

2374 independent reflections

1717 reflections with $I>2 \sigma(I)$

$R_{\text {int }}=0.029$

$\theta_{\max }=25.0^{\circ}, \theta_{\min }=2.7^{\circ}$

$h=-9 \rightarrow 9$

$k=-10 \rightarrow 10$

$l=-13 \rightarrow 13$

Hydrogen site location: mixed

$\mathrm{H}$ atoms treated by a mixture of independent and constrained refinement

$w=1 /\left[\sigma^{2}\left(F_{\mathrm{o}}^{2}\right)+(0.0452 P)^{2}+0.5934 P\right]$

where $P=\left(F_{\mathrm{o}}{ }^{2}+2 F_{\mathrm{c}}{ }^{2}\right) / 3$

$(\Delta / \sigma)_{\max }<0.001$

$\Delta \rho_{\max }=0.24 \mathrm{e} \AA^{-3}$

$\Delta \rho_{\min }=-0.25$ e $\AA^{-3}$ 


\section{Special details}

Geometry. All esds (except the esd in the dihedral angle between two 1.s. planes) are estimated using the full covariance matrix. The cell esds are taken into account individually in the estimation of esds in distances, angles and torsion angles; correlations between esds in cell parameters are only used when they are defined by crystal symmetry. An approximate (isotropic) treatment of cell esds is used for estimating esds involving l.s. planes.

Fractional atomic coordinates and isotropic or equivalent isotropic displacement parameters $\left(\AA^{2}\right)$

\begin{tabular}{|c|c|c|c|c|}
\hline & $x$ & $y$ & $z$ & $U_{\text {iso }} * / U_{\text {eq }}$ \\
\hline $\mathrm{C} 1$ & $0.6227(3)$ & $0.5359(3)$ & $0.3281(2)$ & $0.0338(5)$ \\
\hline $\mathrm{C} 2$ & $0.8010(3)$ & $0.6555(3)$ & $0.4224(2)$ & $0.0342(5)$ \\
\hline $\mathrm{C} 3$ & $0.8216(3)$ & $0.7875(3)$ & $0.5161(2)$ & $0.0371(6)$ \\
\hline $\mathrm{H} 3$ & 0.9405 & 0.8616 & 0.5755 & $0.044^{*}$ \\
\hline $\mathrm{C} 4$ & $0.6675(3)$ & $0.8101(3)$ & $0.5224(2)$ & $0.0356(6)$ \\
\hline $\mathrm{C} 5$ & $0.4892(3)$ & $0.7045(3)$ & $0.4328(2)$ & $0.0352(6)$ \\
\hline H5 & 0.3854 & 0.7222 & 0.4356 & $0.042 *$ \\
\hline C6 & $0.4730(3)$ & $0.5752(3)$ & $0.3417(2)$ & $0.0320(5)$ \\
\hline $\mathrm{C} 7$ & $0.7112(4)$ & 0.0619 (4) & $0.1648(3)$ & $0.0537(7)$ \\
\hline H7 & 0.6806 & 0.0272 & 0.2355 & $0.064^{*}$ \\
\hline $\mathrm{C} 8$ & $0.7330(4)$ & $-0.0490(4)$ & $0.0772(2)$ & $0.0441(6)$ \\
\hline C9 & $0.7774(4)$ & $0.0108(4)$ & $-0.0253(2)$ & $0.0460(7)$ \\
\hline H9 & 0.7911 & -0.0610 & -0.0875 & $0.055^{*}$ \\
\hline $\mathrm{C} 10$ & $0.8018(4)$ & $0.1739(4)$ & $-0.0378(3)$ & $0.0516(7)$ \\
\hline H10 & 0.8335 & 0.2132 & -0.1071 & $0.062 *$ \\
\hline C11 & $0.7792(4)$ & $0.2771(4)$ & $0.0525(3)$ & $0.0530(7)$ \\
\hline H11 & 0.7960 & 0.3885 & 0.0460 & $0.064^{*}$ \\
\hline C12 & $0.7062(5)$ & $-0.2262(4)$ & 0.0915 (3) & $0.0683(9)$ \\
\hline $\mathrm{H} 12 \mathrm{~A}$ & 0.7274 & -0.2835 & 0.0214 & $0.102^{*}$ \\
\hline H12B & 0.7972 & -0.2084 & 0.1665 & $0.102 *$ \\
\hline $\mathrm{H} 12 \mathrm{C}$ & 0.5769 & -0.3007 & 0.0957 & $0.102 *$ \\
\hline N1 & $0.9729(3)$ & 0.6445 (3) & $0.4219(2)$ & $0.0495(6)$ \\
\hline $\mathrm{N} 2$ & $0.6903(3)$ & 0.9444 (3) & $0.6245(2)$ & $0.0461(6)$ \\
\hline N3 & $0.2906(3)$ & $0.4686(3)$ & 0.24334 (19) & $0.0398(5)$ \\
\hline N4 & $0.7332(3)$ & $0.2180(4)$ & $0.1493(2)$ & 0.0549 (7) \\
\hline $\mathrm{O} 1$ & $0.9718(3)$ & $0.5443(3)$ & $0.3360(3)$ & $0.0885(9)$ \\
\hline $\mathrm{O} 2$ & $1.1190(3)$ & 0.7419 (4) & $0.5074(2)$ & $0.0792(8)$ \\
\hline $\mathrm{O} 3$ & $0.8528(3)$ & $1.0446(3)$ & $0.69676(19)$ & $0.0630(6)$ \\
\hline $\mathrm{O} 4$ & $0.5489(3)$ & $0.9540(3)$ & $0.6359(2)$ & $0.0670(6)$ \\
\hline O5 & $0.2505(3)$ & $0.5433(3)$ & $0.1711(2)$ & $0.0799(7)$ \\
\hline O6 & 0.1909 (3) & $0.3122(3)$ & $0.2365(2)$ & $0.0673(6)$ \\
\hline O7 & $0.5902(3)$ & $0.4101(3)$ & $0.24116(17)$ & $0.0518(5)$ \\
\hline $\mathrm{H} 4 \mathrm{~A}$ & $0.712(6)$ & $0.291(5)$ & 0.205 (4) & $0.093(12)^{*}$ \\
\hline
\end{tabular}

Atomic displacement parameters $\left(\AA^{2}\right)$

\begin{tabular}{lllllll}
\hline & $U^{11}$ & $U^{22}$ & $U^{33}$ & $U^{12}$ & $U^{13}$ & $U^{23}$ \\
\hline $\mathrm{C} 1$ & $0.0374(13)$ & $0.0393(13)$ & $0.0313(12)$ & $0.0242(11)$ & $0.0115(10)$ & $0.0080(10)$ \\
$\mathrm{C} 2$ & $0.0287(12)$ & $0.0393(13)$ & $0.0389(13)$ & $0.0200(10)$ & $0.0124(10)$ & $0.0079(10)$
\end{tabular}


supporting information

$\begin{array}{lllllll}\text { C3 } & 0.0301(13) & 0.0386(13) & 0.0358(13) & 0.0136(10) & 0.0085(10) & 0.0050(10) \\ \text { C4 } & 0.0384(14) & 0.0339(12) & 0.0345(12) & 0.0186(11) & 0.0138(11) & 0.0031(10) \\ \text { C5 } & 0.0361(13) & 0.0396(13) & 0.0381(13) & 0.0246(11) & 0.0141(11) & 0.0084(11) \\ \text { C6 } & 0.0310(12) & 0.0372(12) & 0.0302(12) & 0.0203(10) & 0.0074(10) & 0.0063(10) \\ \text { C7 } & 0.0408(16) & 0.072(2) & 0.0438(15) & 0.0268(14) & 0.0152(12) & 0.0057(14) \\ \text { C8 } & 0.0328(13) & 0.0488(15) & 0.0427(15) & 0.0188(12) & 0.0067(11) & 0.0002(12) \\ \text { C9 } & 0.0453(15) & 0.0493(15) & 0.0403(14) & 0.0272(13) & 0.0078(12) & -0.0050(12) \\ \text { C10 } & 0.0584(18) & 0.0547(17) & 0.0434(15) & 0.0330(14) & 0.0112(13) & 0.0048(13) \\ \text { C11 } & 0.0496(17) & 0.0504(16) & 0.0557(18) & 0.0304(14) & 0.0038(14) & -0.0005(14) \\ \text { C12 } & 0.067(2) & 0.062(2) & 0.076(2) & 0.0307(17) & 0.0222(18) & 0.0207(17) \\ \text { N1 } & 0.0350(13) & 0.0548(14) & 0.0596(15) & 0.0254(11) & 0.0136(11) & 0.0064(12) \\ \text { N2 } & 0.0503(14) & 0.0424(12) & 0.0428(12) & 0.0233(11) & 0.0148(11) & 0.0009(10) \\ \text { N3 } & 0.0386(12) & 0.0480(13) & 0.0360(11) & 0.0269(11) & 0.0076(9) & 0.0048(10) \\ \text { N4 } & 0.0416(13) & 0.0639(16) & 0.0530(15) & 0.0316(12) & 0.0076(11) & -0.0137(13) \\ \text { O1 } & 0.0459(13) & 0.0855(16) & 0.114(2) & 0.0365(12) & 0.0156(13) & -0.0352(15) \\ \text { O2 } & 0.0415(12) & 0.116(2) & 0.0693(15) & 0.0458(13) & -0.0011(11) & -0.0092(14) \\ \text { O3 } & 0.0572(13) & 0.0555(12) & 0.0524(12) & 0.0195(10) & 0.0083(10) & -0.0134(10) \\ \text { O4 } & 0.0646(14) & 0.0736(14) & 0.0663(14) & 0.0430(12) & 0.0233(11) & -0.0078(11) \\ \text { O5 } & 0.0700(16) & 0.0835(16) & 0.0718(15) & 0.0343(13) & -0.0089(12) & 0.0310(13) \\ \text { O6 } & 0.0546(13) & 0.0478(13) & 0.0684(14) & 0.0122(10) & -0.0058(10) & 0.0049(10) \\ \text { O7 } & 0.0504(11) & 0.0585(12) & 0.0472(11) & 0.0367(10) & 0.0068(9) & -0.0093(9)\end{array}$

Geometric parameters $\left(\AA,{ }^{\circ}\right)$

\begin{tabular}{llll}
\hline $\mathrm{C} 1-\mathrm{O} 7$ & $1.251(3)$ & $\mathrm{C} 9-\mathrm{C} 10$ & $1.371(4)$ \\
$\mathrm{C} 1-\mathrm{C} 2$ & $1.432(3)$ & $\mathrm{C} 9-\mathrm{H} 9$ & 0.9300 \\
$\mathrm{C} 1-\mathrm{C} 6$ & $1.434(3)$ & $\mathrm{C} 10-\mathrm{C} 11$ & $1.357(4)$ \\
$\mathrm{C} 2-\mathrm{C} 3$ & $1.372(3)$ & $\mathrm{C} 10-\mathrm{H} 10$ & 0.9300 \\
$\mathrm{C} 2-\mathrm{N} 1$ & $1.444(3)$ & $\mathrm{C} 11-\mathrm{N} 4$ & $1.318(4)$ \\
$\mathrm{C} 3-\mathrm{C} 4$ & $1.366(3)$ & $\mathrm{C} 11-\mathrm{H} 11$ & 0.9300 \\
$\mathrm{C} 3-\mathrm{H} 3$ & 0.9300 & $\mathrm{C} 12-\mathrm{H} 12 \mathrm{~A}$ & 0.9600 \\
$\mathrm{C} 4-\mathrm{C} 5$ & $1.394(3)$ & $\mathrm{C} 12-\mathrm{H} 12 \mathrm{~B}$ & 0.9600 \\
$\mathrm{C} 4-\mathrm{N} 2$ & $1.439(3)$ & $\mathrm{C} 12-\mathrm{H} 12 \mathrm{C}$ & 0.9600 \\
$\mathrm{C} 5-\mathrm{C} 6$ & $1.351(3)$ & $\mathrm{N} 1-\mathrm{O} 1$ & $1.205(3)$ \\
$\mathrm{C} 5-\mathrm{H} 5$ & 0.9300 & $\mathrm{~N} 1-\mathrm{O} 2$ & $1.218(3)$ \\
$\mathrm{C} 6-\mathrm{N} 3$ & $1.461(3)$ & $\mathrm{N} 2-\mathrm{O} 4$ & $1.217(3)$ \\
$\mathrm{C} 7-\mathrm{N} 4$ & $1.326(4)$ & $\mathrm{N} 3-\mathrm{O} 3$ & $1.230(3)$ \\
$\mathrm{C} 7-\mathrm{C} 8$ & $1.375(4)$ & $\mathrm{N} 3-\mathrm{O} 5$ & $1.199(3)$ \\
$\mathrm{C} 7-\mathrm{H} 7$ & 0.9300 & $\mathrm{~N} 4-\mathrm{H} 4 \mathrm{~A}$ & $0.93(4)$ \\
$\mathrm{C} 8-\mathrm{C} 9$ & $1.377(4)$ & & \\
$\mathrm{C} 8-\mathrm{C} 12$ & $1.491(4)$ & $\mathrm{C} 8-\mathrm{C} 9-\mathrm{H} 9$ & 119.3 \\
& & $\mathrm{C} 11-\mathrm{C} 10-\mathrm{C} 9$ & $119.0(3)$ \\
$\mathrm{O} 7-\mathrm{C} 1-\mathrm{C} 2$ & $\mathrm{C} 11-\mathrm{C} 10-\mathrm{H} 10$ & 120.5 \\
$\mathrm{O} 7-\mathrm{C} 1-\mathrm{C} 6$ & $127.4(2)$ & $\mathrm{C} 9-\mathrm{C} 10-\mathrm{H} 10$ & 120.5 \\
$\mathrm{C} 2-\mathrm{C} 1-\mathrm{C} 6$ & $120.7(2)$ & $\mathrm{N} 4-\mathrm{C} 11-\mathrm{C} 10$ & $119.4(3)$ \\
$\mathrm{C} 3-\mathrm{C} 2-\mathrm{C} 1$ & $111.90(19)$ & $\mathrm{N} 4-\mathrm{C} 11-\mathrm{H} 11$ & 120.3 \\
$\mathrm{C} 3-\mathrm{C} 2-\mathrm{N} 1$ & $123.2(2)$ & & \\
$\mathrm{C} 1-\mathrm{C} 2-\mathrm{N} 1$ & $116.0(2)$ & $120.8(2)$ &
\end{tabular}




\begin{tabular}{|c|c|c|c|}
\hline $\mathrm{C} 4-\mathrm{C} 3-\mathrm{C} 2$ & $120.0(2)$ & $\mathrm{C} 10-\mathrm{C} 11-\mathrm{H} 11$ & 120.3 \\
\hline $\mathrm{C} 4-\mathrm{C} 3-\mathrm{H} 3$ & 120.0 & $\mathrm{C} 8-\mathrm{C} 12-\mathrm{H} 12 \mathrm{~A}$ & 109.5 \\
\hline $\mathrm{C} 2-\mathrm{C} 3-\mathrm{H} 3$ & 120.0 & $\mathrm{C} 8-\mathrm{C} 12-\mathrm{H} 12 \mathrm{~B}$ & 109.5 \\
\hline $\mathrm{C} 3-\mathrm{C} 4-\mathrm{C} 5$ & $121.2(2)$ & $\mathrm{H} 12 \mathrm{~A}-\mathrm{C} 12-\mathrm{H} 12 \mathrm{~B}$ & 109.5 \\
\hline $\mathrm{C} 3-\mathrm{C} 4-\mathrm{N} 2$ & $119.0(2)$ & $\mathrm{C} 8-\mathrm{C} 12-\mathrm{H} 12 \mathrm{C}$ & 109.5 \\
\hline $\mathrm{C} 5-\mathrm{C} 4-\mathrm{N} 2$ & $119.7(2)$ & $\mathrm{H} 12 \mathrm{~A}-\mathrm{C} 12-\mathrm{H} 12 \mathrm{C}$ & 109.5 \\
\hline $\mathrm{C} 6-\mathrm{C} 5-\mathrm{C} 4$ & $117.4(2)$ & $\mathrm{H} 12 \mathrm{~B}-\mathrm{C} 12-\mathrm{H} 12 \mathrm{C}$ & 109.5 \\
\hline $\mathrm{C} 6-\mathrm{C} 5-\mathrm{H} 5$ & 121.3 & $\mathrm{O} 1-\mathrm{N} 1-\mathrm{O} 2$ & $121.1(2)$ \\
\hline $\mathrm{C} 4-\mathrm{C} 5-\mathrm{H} 5$ & 121.3 & $\mathrm{O} 1-\mathrm{N} 1-\mathrm{C} 2$ & $120.0(2)$ \\
\hline $\mathrm{C} 5-\mathrm{C} 6-\mathrm{C} 1$ & $126.1(2)$ & $\mathrm{O} 2-\mathrm{N} 1-\mathrm{C} 2$ & $118.8(2)$ \\
\hline $\mathrm{C} 5-\mathrm{C} 6-\mathrm{N} 3$ & $118.5(2)$ & $\mathrm{O} 4-\mathrm{N} 2-\mathrm{O} 3$ & $122.8(2)$ \\
\hline $\mathrm{C} 1-\mathrm{C} 6-\mathrm{N} 3$ & $115.36(19)$ & $\mathrm{O} 4-\mathrm{N} 2-\mathrm{C} 4$ & $119.0(2)$ \\
\hline $\mathrm{N} 4-\mathrm{C} 7-\mathrm{C} 8$ & $120.7(3)$ & $\mathrm{O} 3-\mathrm{N} 2-\mathrm{C} 4$ & $118.2(2)$ \\
\hline $\mathrm{N} 4-\mathrm{C} 7-\mathrm{H} 7$ & 119.6 & $\mathrm{O} 6-\mathrm{N} 3-\mathrm{O} 5$ & $123.3(2)$ \\
\hline $\mathrm{C} 8-\mathrm{C} 7-\mathrm{H} 7$ & 119.6 & $\mathrm{O} 6-\mathrm{N} 3-\mathrm{C} 6$ & $119.3(2)$ \\
\hline $\mathrm{C} 7-\mathrm{C} 8-\mathrm{C} 9$ & $116.5(3)$ & $\mathrm{O} 5-\mathrm{N} 3-\mathrm{C} 6$ & $117.4(2)$ \\
\hline $\mathrm{C} 7-\mathrm{C} 8-\mathrm{C} 12$ & $121.8(3)$ & $\mathrm{C} 11-\mathrm{N} 4-\mathrm{C} 7$ & $123.0(2)$ \\
\hline $\mathrm{C} 9-\mathrm{C} 8-\mathrm{C} 12$ & $121.8(2)$ & $\mathrm{C} 11-\mathrm{N} 4-\mathrm{H} 4 \mathrm{~A}$ & $115(2)$ \\
\hline $\mathrm{C} 10-\mathrm{C} 9-\mathrm{C} 8$ & $121.5(2)$ & $\mathrm{C} 7-\mathrm{N} 4-\mathrm{H} 4 \mathrm{~A}$ & $122(2)$ \\
\hline $\mathrm{C} 10-\mathrm{C} 9-\mathrm{H} 9$ & 119.3 & & \\
\hline $\mathrm{O} 7-\mathrm{C} 1-\mathrm{C} 2-\mathrm{C} 3$ & $-178.4(2)$ & $\mathrm{C} 7-\mathrm{C} 8-\mathrm{C} 9-\mathrm{C} 10$ & $1.2(4)$ \\
\hline $\mathrm{C} 6-\mathrm{C} 1-\mathrm{C} 2-\mathrm{C} 3$ & $1.9(3)$ & $\mathrm{C} 12-\mathrm{C} 8-\mathrm{C} 9-\mathrm{C} 10$ & $-180.0(3)$ \\
\hline $\mathrm{O} 7-\mathrm{C} 1-\mathrm{C} 2-\mathrm{N} 1$ & $2.7(4)$ & $\mathrm{C} 8-\mathrm{C} 9-\mathrm{C} 10-\mathrm{C} 11$ & $-0.8(4)$ \\
\hline $\mathrm{C} 6-\mathrm{C} 1-\mathrm{C} 2-\mathrm{N} 1$ & $-177.0(2)$ & $\mathrm{C} 9-\mathrm{C} 10-\mathrm{C} 11-\mathrm{N} 4$ & $-0.4(4)$ \\
\hline $\mathrm{C} 1-\mathrm{C} 2-\mathrm{C} 3-\mathrm{C} 4$ & $-0.2(4)$ & $\mathrm{C} 3-\mathrm{C} 2-\mathrm{N} 1-\mathrm{O} 1$ & $-172.7(3)$ \\
\hline $\mathrm{N} 1-\mathrm{C} 2-\mathrm{C} 3-\mathrm{C} 4$ & $178.7(2)$ & $\mathrm{C} 1-\mathrm{C} 2-\mathrm{N} 1-\mathrm{O} 1$ & $6.3(4)$ \\
\hline $\mathrm{C} 2-\mathrm{C} 3-\mathrm{C} 4-\mathrm{C} 5$ & $-1.9(4)$ & $\mathrm{C} 3-\mathrm{C} 2-\mathrm{N} 1-\mathrm{O} 2$ & $5.0(4)$ \\
\hline $\mathrm{C} 2-\mathrm{C} 3-\mathrm{C} 4-\mathrm{N} 2$ & $177.4(2)$ & $\mathrm{C} 1-\mathrm{C} 2-\mathrm{N} 1-\mathrm{O} 2$ & $-176.0(3)$ \\
\hline $\mathrm{C} 3-\mathrm{C} 4-\mathrm{C} 5-\mathrm{C} 6$ & $2.0(4)$ & $\mathrm{C} 3-\mathrm{C} 4-\mathrm{N} 2-\mathrm{O} 4$ & $-173.7(2)$ \\
\hline $\mathrm{N} 2-\mathrm{C} 4-\mathrm{C} 5-\mathrm{C} 6$ & $-177.3(2)$ & $\mathrm{C} 5-\mathrm{C} 4-\mathrm{N} 2-\mathrm{O} 4$ & $5.6(4)$ \\
\hline $\mathrm{C} 4-\mathrm{C} 5-\mathrm{C} 6-\mathrm{C} 1$ & $0.0(4)$ & $\mathrm{C} 3-\mathrm{C} 4-\mathrm{N} 2-\mathrm{O} 3$ & $6.1(4)$ \\
\hline $\mathrm{C} 4-\mathrm{C} 5-\mathrm{C} 6-\mathrm{N} 3$ & $-177.0(2)$ & $\mathrm{C} 5-\mathrm{C} 4-\mathrm{N} 2-\mathrm{O} 3$ & $-174.6(2)$ \\
\hline $\mathrm{O} 7-\mathrm{C} 1-\mathrm{C} 6-\mathrm{C} 5$ & $178.5(2)$ & $\mathrm{C} 5-\mathrm{C} 6-\mathrm{N} 3-\mathrm{O} 6$ & $-112.1(3)$ \\
\hline $\mathrm{C} 2-\mathrm{C} 1-\mathrm{C} 6-\mathrm{C} 5$ & $-1.8(3)$ & $\mathrm{C} 1-\mathrm{C} 6-\mathrm{N} 3-\mathrm{O} 6$ & $70.5(3)$ \\
\hline $\mathrm{O} 7-\mathrm{C} 1-\mathrm{C} 6-\mathrm{N} 3$ & $-4.4(3)$ & $\mathrm{C} 5-\mathrm{C} 6-\mathrm{N} 3-\mathrm{O} 5$ & $69.4(3)$ \\
\hline $\mathrm{C} 2-\mathrm{C} 1-\mathrm{C} 6-\mathrm{N} 3$ & $175.3(2)$ & $\mathrm{C} 1-\mathrm{C} 6-\mathrm{N} 3-\mathrm{O} 5$ & $-108.0(3)$ \\
\hline $\mathrm{N} 4-\mathrm{C} 7-\mathrm{C} 8-\mathrm{C} 9$ & $-0.3(4)$ & $\mathrm{C} 10-\mathrm{C} 11-\mathrm{N} 4-\mathrm{C} 7$ & $1.4(4)$ \\
\hline $\mathrm{N} 4-\mathrm{C} 7-\mathrm{C} 8-\mathrm{C} 12$ & $-179.1(3)$ & $\mathrm{C} 8-\mathrm{C} 7-\mathrm{N} 4-\mathrm{C} 11$ & $-1.0(4)$ \\
\hline
\end{tabular}

Hydrogen-bond geometry $\left(A,{ }^{\circ}\right)$

\begin{tabular}{lllll}
\hline$D-\mathrm{H} \cdots A$ & $D-\mathrm{H}$ & $\mathrm{H} \cdots A$ & $D \cdots A$ & $D-\mathrm{H} \cdots A$ \\
\hline $\mathrm{N} 4-\mathrm{H} 4 A \cdots \mathrm{O} 1$ & $0.93(4)$ & $2.27(4)$ & $2.827(4)$ & $118(4)$ \\
$\mathrm{N} 4-\mathrm{H} 4 A \cdots \mathrm{O} 7$ & $0.93(4)$ & $1.79(5)$ & $2.638(4)$ & $152(4)$ \\
$\mathrm{C} 5-\mathrm{H} 5 \cdots \mathrm{O} 2^{\mathrm{i}}$ & 0.93 & 2.51 & $3.406(4)$ & 162
\end{tabular}


supporting information

$\mathrm{C} 10-\mathrm{H} 10 \cdots \mathrm{O} 3^{\mathrm{ii}}$

$\mathrm{C} 12-\mathrm{H} 12 B^{\cdots} \mathrm{O}^{3}{ }^{\mathrm{iii}}$

Symmetry codes: (i) $x-1, y, z$; (ii) $x, y-1, z^{-1}$; (iii) $-x+2,-y+1,-z+1$.
$3.220(4)$

$3.414(5)$
129

149 